\title{
Investigating vascular diseases in people living with HIV by nuclear imaging
}

\author{
Phillip Chan, ${ }^{\mathrm{a}}$ and Serena Spudich ${ }^{\mathrm{b}}$ \\ a SEARCH, Institute of HIV Research and Innovation, Bangkok, Thailand \\ b Department of Neurology, Yale University School of Medicine, New Haven, CT
}

Received Mar 10, 2021; accepted Mar 11, 2021

doi: $10.1007 / \mathrm{s} 12350-021-02613-x$

\section{See related article, pp. 1566-1575}

In addition to the progressive loss of adaptive immunity leading to the acquired immunodeficiency syndrome (AIDS), HIV-1 infection impacts almost all bodily systems including the cardiovascular system (CVS) and central nervous system (CNS). Compared to HIV-negative individuals, people living with HIV (PLWH) have about 2-fold increased relative risk (RR) of coronary arterial disease $(\mathrm{CAD})^{1}$ and 3 -fold increased RR of stroke. ${ }^{2}$ Despite initial concern that metabolic toxicity of antiretroviral therapy (ART) may underlie these risks, international randomized controlled trials have confirmed that immediate initiation of non-interrupted ART reduces vascular complications in addition to the restoration of immune function. ${ }^{3}$ In the present era, the life expectancy of PLWH is approaching that of HIV-negative populations. Yet, in this context of longterm survival, the burden of non-communicable diseases in aging populations of PLWH on suppressive ART emerges as a major health care concern. Vascular diseases, especially CAD and stroke, are under the spotlight not only because of their historical appearance in the pre-ART era but also their universal role in morbidity and mortality in all populations. In this issue of $\mathrm{JNC}$, Taglieri et al. compare the arterial inflammation in the ascending and descending aorta using ${ }^{18}$ F-Fluorodeoxyglucose $\left({ }^{18} \mathrm{FDG}\right) \mathrm{PET}$ in a group of PLWH on stable ART.

\footnotetext{
Reprint requests: Serena Spudich, Department of Neurology, Yale University School of Medicine, New Haven, CT; serena.spudich@yale.edu

J Nucl Cardiol 2022;29:1576-82.

$1071-3581 / \$ 34.00$

Copyright (C) 2021 American Society of Nuclear Cardiology.
}

\section{THE BURDEN OF VASCULAR DISEASES AMONG PLWH IN THE ART ERA}

PLWH consistently show a higher risk of cardiovascular disease (CVD) than HIV-negative populations despite stable ART. In a systematic review of 80 longitudinal CVD studies with 793,635 PLWH, the global burden of HIV-associated CVD has tripled over the past 2 decades. ${ }^{4}$ PLWH presenting with the acute coronary syndrome (ACS) are 10 years younger than HIV-negative patients. ${ }^{5}$ In a nation-wide, retrospective Danish study, PLWH had an increased RR of 1.6 of developing a cerebrovascular event than HIV-negative individuals after controlling for intravenous drug use and other traditional vascular risk factors. ${ }^{6}$ A modeling study of 8791 treated Dutch PLWH predicted that annual CVD incidence and costs will increase by $55 \%$ and 36\%, respectively, between 2015 and $2030 .^{7}$ Understanding the interaction and between HIV and atherosclerosis, as well the management, is thus crucial to maintain long-term quality of life and resilient aging of PLWH.

\section{ATHEROSCLEROSIS IN PLWH ON SUPPRESSIVE ART}

HIV appears to alter the course and presentation of atherosclerosis and CAD. Compared to Type I ACS that are the predominant cardiac events in HIV-negative populations, up to $50 \%$ of myocardial infarctions in PLWH are Type II ACS ${ }^{8}$ attributed to a mismatch of oxygen demand and supply in PLWH. ${ }^{9}$ Structurally, non-calcified coronary plaque is more prevalent in PLWH than in HIV-negative individuals. ${ }^{10} \mathrm{~A}$ number of persistent phenomena may promote atherosclerosis in PLWH despite suppressive ART. Systemic immune dysregulation, including monocyte/macrophage activation and T-cell dysregulation, persists in PLWH on suppressive ART. ${ }^{11}$ Increased markers of monocyte/macrophage activation, such as plasma sCD14 
and SCD163, associate with atherosclerosis in both HIVpositive and HIV-negative individuals. ${ }^{12}$ These markers remain elevated among PLWH despite HIV suppression. ${ }^{12}$ Low CD4/CD8 ratio, a marker of T-cell dysregulation and immunosenescence, remains prevalent in PLWH despite plasma HIV suppression ${ }^{13,14}$ and is associated with CAD events in PLWH. ${ }^{15}$

HIV reservoir may play a role in atherosclerosis. HIV reservoir is still able to produce HIV-encoded proteins including transactivator of transcription (Tat), negative factor (Nef), and envelope protein gp120 through low-level transcription during plasma viral suppression. ${ }^{16,17}$ These proteins are linked to inflammation, endothelial dysfunction, and endothelin 1 production. ${ }^{18}$ Increased microbial translocation in the gut, currently considered as an important contributor of atherosclerosis, ${ }^{19}$ persists among PLWH on suppressive ART.

Compared to HIV-negative populations, PLWH also have higher rate of cytomegalovirus (CMV) and Hepatitis $\mathrm{C}$ virus $(\mathrm{HCV})$ co-infections that are linked with atherosclerosis and CVD. ${ }^{20,21}$ Commonly used antiretroviral agents, such as protease Inhibitors, are associated with dyslipidemia that worsens atherosclerosis. Most importantly, PLWH on suppressive ART persistently show metabolic dysfunction in glucose and lipid metabolism. ${ }^{22}$ As a result, HIV plays both direct and indirect roles in atherosclerosis.

\section{ADVANTAGES OF NUCLEAR IMAGING IN ASSESSING ATHEROSCLEROSIS IN HIV}

Given the fact that augmented inflammation is likely the central mechanism of HIV-related atherosclerosis, conventional vascular imaging may be limited by its focus on assessment of structural changes, underestimating inflammatory processes. Despite this, even conventional vascular imaging can reveal distinctions between PLWH and HIV-negative comparison patients. In a prospective study of 811 men and 1011 women from the Multicenter AIDS Cohort Study and the Women's Interagency HIV Study, participants with HIV on ART had increased plaque at common carotid artery detected by repeated B-mode ultrasound imaging than HIV-negative individuals. ${ }^{23}$ In another study that examined the progression of subclinical atherosclerosis over 2 years by coronary artery calcium scan and coronary computed tomography angiography, Framingham risk score (FRS) but not HIV infection was associated with progression of subclinical atherosclerosis. ${ }^{24}$ Yet, the HIV-positive group in the latter study had a similar FRS as the HIV-negative control group despite a younger age, highlighting again the complex relationship between HIV, traditional risk factors, and atherosclerosis.

In the last decade, vascular PET imaging has emerged as a promising tool for risk stratification for atherosclerotic vascular disease. Early studies confirmed the capability of FDG-PET to evaluate the risk of carotid atheroma that leads to recurrent ischemic stroke. ${ }^{25}$ The close association between FDG uptake and CD68+ macrophage burden in plaques further supports its usefulness to evaluate vascular inflammation, ${ }^{26}$ making FDG-PET a potentially superior tool for evaluating HIV-related atherosclerosis compared to conventional imaging such as CT angiogram and ultrasound. A number of nuclear imaging studies focused on HIVrelated atherosclerosis have been reported in the last decade (Table 1). In an early FDG-PET study that examined the effect of ART in 12 treatment-naïve PLWH, aortic FDG uptake did not show significant change 6 months after ART initiation. ${ }^{27}$ The result suggests that vascular inflammation is not readily reverted in the initial months post ART, while the long-term benefit of suppressive ART in atherosclerosis remains less clear. Titanji et al. recently reviewed the outcomes of intervention studies that aimed to alleviate cardiovascular inflammation in HIV. ${ }^{28}$ Of note, atorvastatin, a lipid-lowering agent, only reduces non-calcified plaque volume and high-risk coronary plaque features in PLWH without reducing ${ }^{18}$ FDG uptake within the aorta. ${ }^{29}$ However, subcutaneous administration of canakinumab, a IL-1ß inhibitor, results in reduction in both aortic TBR and plasma inflammatory markers. ${ }^{30}$

\section{EVALUATING ATHEROSCLEROSIS IN PLWH ON SUPPRESSIVE ART BY NUCLEAR IMAGING}

The work from Taglieri et al. provides insight on atherosclerosis in PLWH on long-term suppression. The team compared the arterial inflammation (AI), denoted by the maximum target-to-background ratio (TBRmax) in ${ }^{18}$ FDG PET at the ascending and descending aorta and carotid artery between PLWH and uninfected controls with no known cardiovascular diseases but at least 3 traditional cardiovascular risk factors. Of note, the HIV-positive group was well maintained with ART, indicated by the long duration of ART usage, high frequency of plasma viral suppression, and relatively normal median CD4+ T-cell count of 871 cells $/ \mathrm{mm}^{3}$. Despite similar American College of Cardiology/ atherosclerotic cardiovascular disease (ASCVD) scores between the two groups, participants with HIV showed worse TBRmax at the ascending aorta. Further, the HIV status remained independently associated with worse TBRmax. 


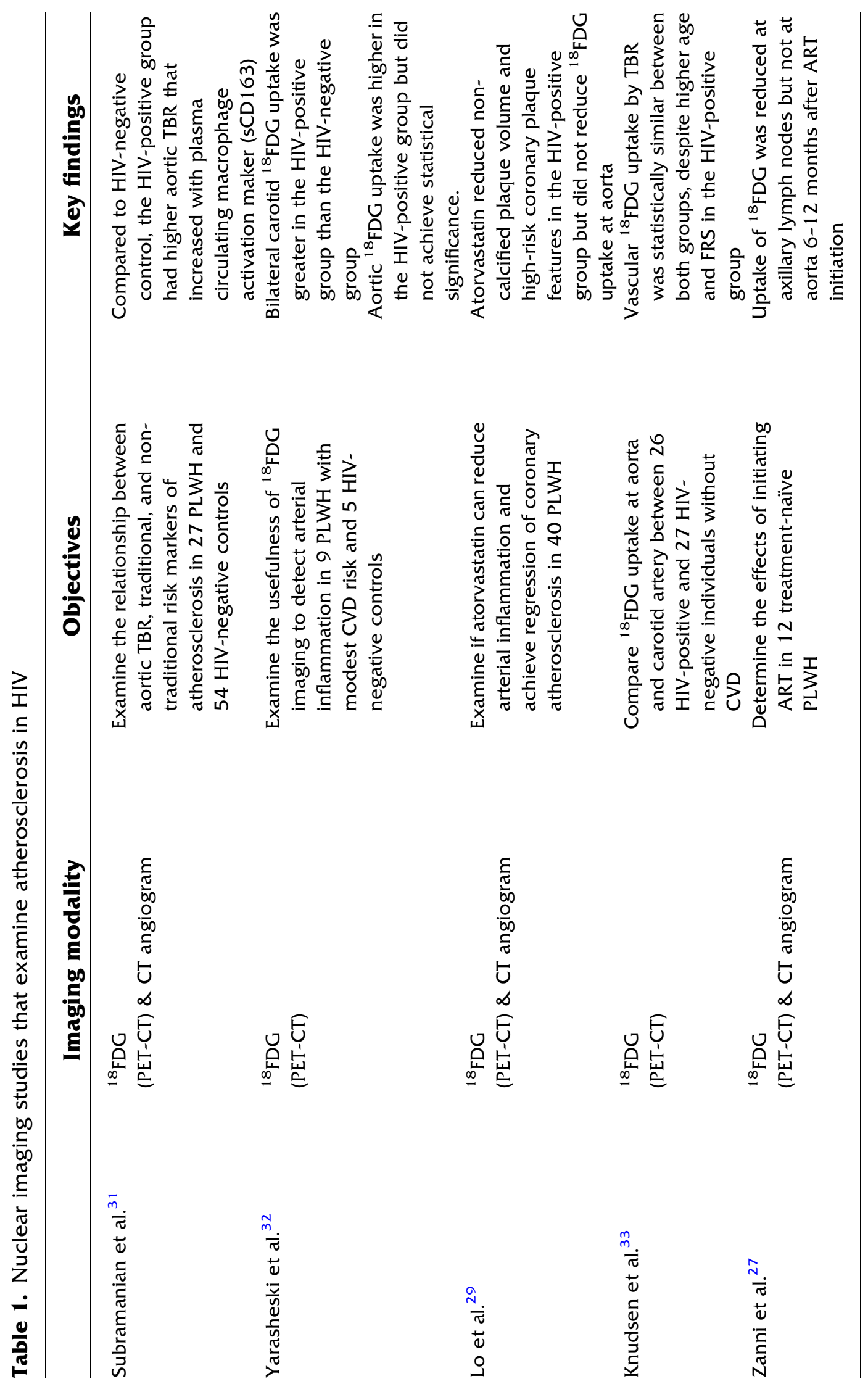




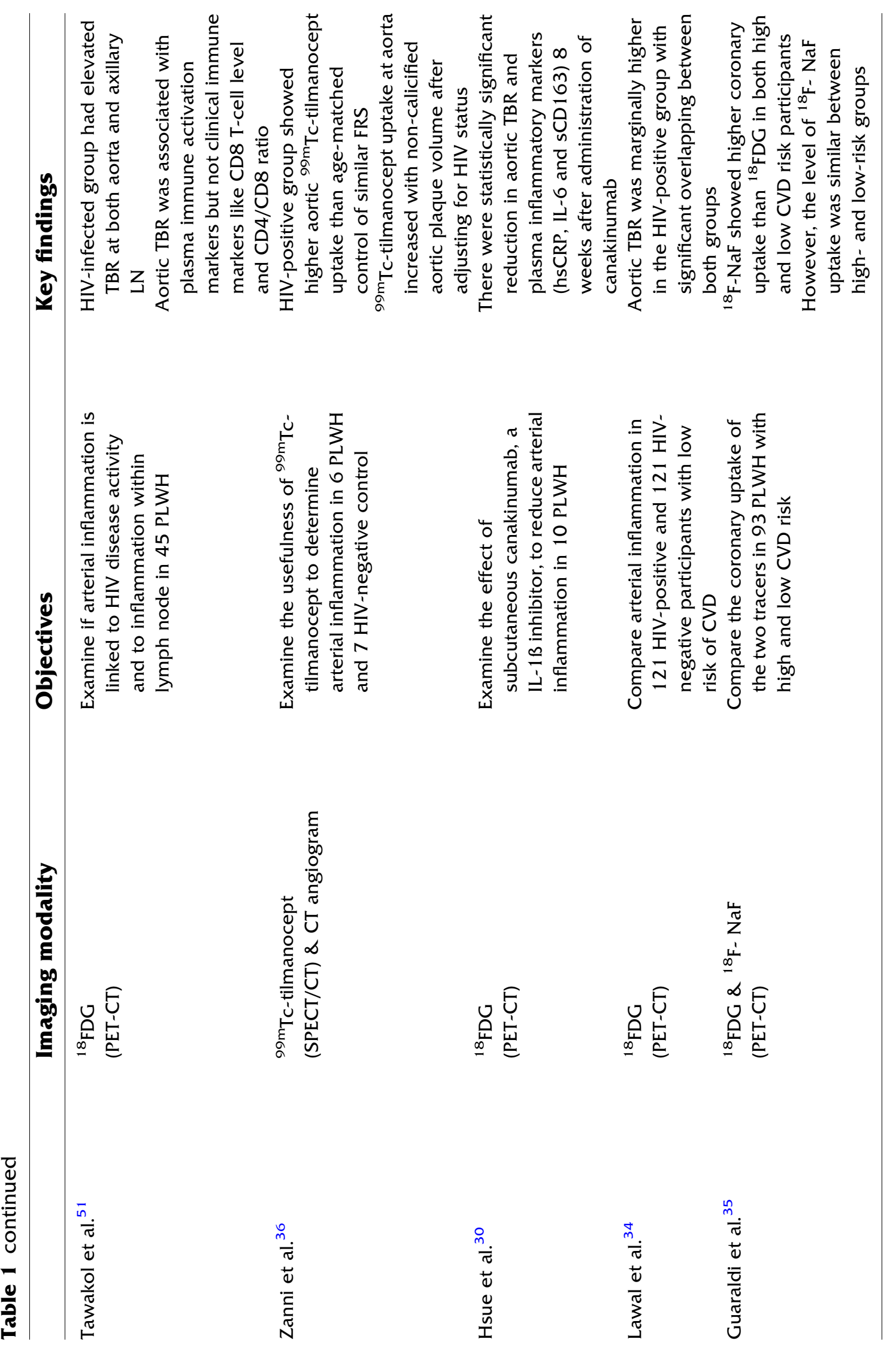


The detection of increased vascular ${ }^{18}$ FDG uptake in this latest work from Taglieri et al. is in line with two early PET studies that compared vascular inflammation between HIV-positive participants on suppressive ART and HIV-negative controls. ${ }^{31,32}$ While both studies detected higher inflammatory signals in participants with HIV, one further study showed an elevated aortic TBR in the group of PLWH in comparison with a FRSmatched HIV-negative control. ${ }^{31}$ However, the difference in vascular FDG uptake by HIV status is less distinctive in young individuals with low CVD risk..$^{33,34}$ Taken together, the findings highlight that elevated atherosclerotic inflammation persists years after ART initiation and viral control. Additionally, applying traditional CVD risk stratification methods in PLWH, especially in those of moderate-to-high CVD risk, may underestimate the actual risk by overlooking the impact of HIV-related inflammation in atherosclerosis. More recently, radiomarkers including ${ }^{18} \mathrm{~F}-\mathrm{NaF}$ and ${ }^{99 \mathrm{~m}} \mathrm{Tc}-$ tilmanocept have been tested for their validity in assessing HIV-related atherosclerosis. Compared to ${ }^{18} \mathrm{FDG},{ }^{18} \mathrm{~F}-\mathrm{NaF}$ appears to have better uptake at the vascular wall but lacks sensitivity to differentiate between high- and low-risk CVD. ${ }^{35}{ }^{99 m}$ Tc-tilmanocept is a radiomarker with CD206+ macrophage binding capacity. In a single-photon emission computed tomography/computed tomography (SPECT/CT) study, ${ }^{99 \mathrm{~m}} \mathrm{Tc}-$ tilmanocept uptake is correlated with non-calicified aortic plaque volume and immunological markers including sCD14 level, absolute number of CD14+CD16- monocytes, and absolute CD8+ T-cell count. $^{36}$

\section{APPLICATION OF NUCLEAR IMAGING TO UNDERSTAND NEUROHIV}

Nuclear imaging is similarly useful in neuroHIV research. Apart from CNS opportunistic infections secondary to overt immunodeficiency, HIV directly invades the CNS and eventually leads to different degrees of cognitive impairment in untreated PLWH. Epidemiology studies reveal that HIV-associated dementia, the most severe type of HIV-associated neurocognitive disorder (HAND), has become uncommon in the ART era. ${ }^{37}$ However, milder forms of HAND persist at a prevalence ranging from $30-50 \%$ of PLWH. While residual neurological deficits resulting from preART neurologic injury could account for part of the persistence of HAND in the ART era, CSF markers and MR spectroscopy studies confirm persistent cerebral inflammation in PLWH on suppressive ART. ${ }^{38}$ Brain PET studies based on macrophage/microglia-specific ligands further reveal that PLWH on suppressive ART with worse cognitive performance have increased marker uptake in the brain, ${ }^{39-41}$ highlighting the association between cognitive impairment and persistent cerebral inflammation.

Several hypotheses have been proposed for the persistence of HAND despite ART, including whether persistent cerebral inflammation might accelerate or augment the manifestation of underlying neurodegenerative diseases such as Alzheimer's disease (AD). This hypothesis is supported by the alterations of CSF AD biomarkers including beta-amyloid and Tau levels in PLWH with cognitive impairment. ${ }^{42,43}$ However, recent PET studies do not show major differences in amyloid deposition stratified by HIV serostatus or HAND severity. ${ }^{44,45}$ Others propose that cognitive impairment in PLWH in the ART era might be related to cerebrovascular diseases. ${ }^{46}$ Asymptomatic intracranial atherosclerosis has been increasingly recognized as a risk factor of cognitive impairment in the general population. ${ }^{47} \mathrm{~A}$ recent post-mortem study demonstrates preclinical atherosclerotic changes of small-to-mediumsized intracranial arteries in PLWH within the first 6 years of HIV infection, ${ }^{48}$ suggesting that ART reduces inflammation but does not resolve arterial remodeling. ${ }^{48}$ To date, longitudinal studies that investigate the impact of atherosclerosis on cognitive function in PLWH are not available.

In summary, persistent systemic immune activation in PLWH on suppressive ART likely continuously fuels the atherosclerosis process. PET imaging has emerged as an ideal tool to examine HIV-related atherosclerosis as it provides both structural information of plaque and quantitative information of inflammation when it is coadministrated with computed tomography (CT) or magnetic resonance imaging (MRI). With the availability of new PET ligands that target specific biological process or cell types, PET has the potential to serve not only as a tool for research but also as a means for disease and treatment effect monitoring. Longitudinal studies that correlate systemic immune activation, vascular inflammation, and plaque progression would help to clarify the CVD risks of PLWH on long-term suppressive treatment. Future studies should also specifically examine changes in vascular inflammation shortly after ART initiation and concomitant with immune reconstitution, as stroke events appears to be more common during the first year of ART. ${ }^{49}$ Additionally, the role of the HIV reservoir in vascular inflammation should be explored as cell-associated HIV DNA and RNA in CD4+ T-cells are independently associated with progression of carotid intima-media thickness and incident plaque development in PLWH. ${ }^{50}$ Lastly, standardizing the methodology of PET imaging acquisition and evaluation would facilitate multicenter study across populations. 


\section{References}

1. Triant VA. Cardiovascular disease and HIV infection. Curr HIV/ AIDS Rep 2013;10:199-206.

2. Chow FC, Regan S, Feske S, Meigs JB, Grinspoon SK, Triant VA. Comparison of ischemic stroke incidence in HIV-infected and non-HIV-infected patients in a US health care system. J Acquir Immune Defic Syndr 2012;60:351-8.

3. Siedner MJ. START or SMART? Timing of antiretroviral therapy initiation and cardiovascular risk for people with human immunodeficiency virus infection. Open Forum Infect Dis 2016;3: ofw032.

4. Shah ASV, Stelzle D, Lee KK, Beck EJ, Alam S, Clifford S, et al. Global burden of atherosclerotic cardiovascular disease in people living with HIV: Systematic review and meta-analysis. Circulation 2018;138:1100-12.

5. Hsue PY, Giri K, Erickson S, MacGregor JS, Younes N, Shergill $\mathrm{A}$, et al. Clinical features of acute coronary syndromes in patients with human immunodeficiency virus infection. Circulation 2004;109:316-9.

6. Rasmussen LD, Engsig FN, Christensen H, Gerstoft J, Kronborg G, Pedersen C, et al. Risk of cerebrovascular events in persons with and without HIV: A Danish nationwide population-based cohort study. AIDS 2011;25:1637-46.

7. Smit M, van Zoest RA, Nichols BE, Vaartjes I, Smit C, van der Valk M, et al. Cardiovascular disease prevention policy in human immunodeficiency virus: Recommendations from a modeling study. Clin Infect Dis 2018;66:743-50.

8. Crane HM, Paramsothy P, Drozd DR, Nance RM, Delaney JA, Heckbert SR, et al. Types of myocardial infarction among human immunodeficiency virus-infected individuals in the United States. JAMA Cardiol 2017;2:260-7.

9. DeFilippis AP, Chapman AR, Mills NL, de Lemos JA, ArbabZadeh A, Newby LK, et al. Assessment and treatment of patients with type 2 myocardial infarction and acute nonischemic myocardial injury. Circulation 2019;140:1661-78.

10. D'Ascenzo F, Cerrato E, Calcagno A, Grossomarra W, Ballocca F, Omede $\mathrm{P}$, et al. High prevalence at computed coronary tomography of non-calcified plaques in asymptomatic HIV patients treated with HAART: A meta-analysis. Atherosclerosis 2015;240:197204.

11. Hileman CO, Funderburg NT. Inflammation, immune activation, and antiretroviral therapy in HIV. Curr HIV/AIDS Rep 2017;14:93-100.

12. McKibben RA, Margolick JB, Grinspoon S, Li X, Palella FJ, Jr., Kingsley LA, et al. Elevated levels of monocyte activation markers are associated with subclinical atherosclerosis in men with and those without HIV infection. J Infect Dis 2015;211:121928.

13. Mussini C, Lorenzini P, Cozzi-Lepri A, Lapadula G, Marchetti G, Nicastri E, et al. CD4/CD8 ratio normalisation and non-AIDSrelated events in individuals with HIV who achieve viral load suppression with antiretroviral therapy: an observational cohort study. Lancet HIV 2015;2:e98-106.

14. Leung V, Gillis J, Raboud J, Cooper C, Hogg RS, Loutfy MR, et al. Predictors of CD4:CD8 ratio normalization and its effect on health outcomes in the era of combination antiretroviral therapy. PLoS ONE 2013;8:

15. Castilho JL, Shepherd BE, Koethe J, Turner M, Bebawy S, Logan $\mathrm{J}$, et al. CD4+/CD8+ ratio, age, and risk of serious noncommunicable diseases in HIV-infected adults on antiretroviral therapy. AIDS 2016;30:899-908.
16. Imamichi H, Smith $M$, Adelsberger JW, Izumi T, Scrimieri F, Sherman BT, et al. Defective HIV-1 proviruses produce viral proteins. Proc Natl Acad Sci U S A 2020;117:3704-10.

17. DeMaster LK, Liu X, VanBelzen DJ, Trinite B, Zheng L, Agosto LM, et al. A subset of CD4/CD8 double-negative T cells expresses HIV proteins in patients on antiretroviral therapy. J Virol 2015;90:2165-79.

18. Wang T, Yi R, Green LA, Chelvanambi S, Seimetz M, Clauss M. Increased cardiovascular disease risk in the HIV-positive population on ART: potential role of HIV-Nef and Tat. Cardiovasc Pathol 2015;24:279-82.

19. Jonsson AL, Backhed F. Role of gut microbiota in atherosclerosis. Nat Rev Cardiol 2017;14:79-87.

20. Wang H, Peng G, Bai J, He B, Huang K, Hu X, et al. Cytomegalovirus infection and relative risk of cardiovascular disease (ischemic heart disease, stroke, and cardiovascular death): a metaanalysis of prospective studies up to 2016. J Am Heart Assoc $2017 ; 6$.

21. Adinolfi LE, Rinaldi L, Nevola R. Chronic hepatitis C, atherosclerosis and cardiovascular disease: What impact of directacting antiviral treatments? World J Gastroenterol 2018;24:461721.

22. Willig AL, Overton ET. Metabolic complications and glucose metabolism in HIV infection: A review of the evidence. Curr HIV/ AIDS Rep 2016;13:289-96.

23. Hanna DB, Post WS, Deal JA, Hodis HN, Jacobson LP, Mack WJ, et al. HIV infection is associated with progression of subclinical carotid atherosclerosis. Clin Infect Dis 2015;61:640-50.

24. Tarr PE, Ledergerber B, Calmy A, Doco-Lecompte T, Schoepf IC, Marzel A, et al. Longitudinal progression of subclinical coronary atherosclerosis in Swiss HIV-positive compared with HIV-negative persons undergoing coronary calcium score scan and CT angiography. Open Forum Infect Dis 2020;7:438.

25. Marnane M, Merwick A, Sheehan OC, Hannon N, Foran P, Grant $\mathrm{T}$, et al. Carotid plaque inflammation on 18F-fluorodeoxyglucose positron emission tomography predicts early stroke recurrence. Ann Neurol 2012;71:709-18.

26. Tawakol A, Migrino RQ, Bashian GG, Bedri S, Vermylen D, Cury $\mathrm{RC}$, et al. In vivo $18 \mathrm{~F}$-fluorodeoxyglucose positron emission tomography imaging provides a noninvasive measure of carotid plaque inflammation in patients. J Am Coll Cardiol 2006;48:181824.

27. Zanni MV, Toribio M, Robbins GK, Burdo TH, Lu MT, Ishai AE, et al. Effects of antiretroviral therapy on immune function and arterial inflammation in treatment-naive patients with human immunodeficiency virus infection. JAMA Cardiol 2016;1:474-80.

28. Titanji B, Gavegnano C, Hsue P, Schinazi R, Marconi VC. Targeting inflammation to reduce atherosclerotic cardiovascular risk in people with HIV infection. J Am Heart Assoc 2020;9:

29. Lo J, Lu MT, Ihenachor EJ, Wei J, Looby SE, Fitch KV, et al. Effects of statin therapy on coronary artery plaque volume and high-risk plaque morphology in HIV-infected patients with subclinical atherosclerosis: a randomised, double-blind, placebocontrolled trial. Lancet HIV 2015;2:e52-63.

30. Hsue PY, Li D, Ma Y, Ishai A, Manion M, Nahrendorf M, et al. IL-1beta inhibition reduces atherosclerotic inflammation in HIV infection. J Am Coll Cardiol 2018;72:2809-11.

31. Subramanian S, Tawakol A, Burdo TH, Abbara S, Wei J, Vijayakumar $\mathrm{J}$, et al. Arterial inflammation in patients with HIV. JAMA 2012;308:379-86

32. Yarasheski KE, Laciny E, Overton ET, Reeds DN, Harrod M, Baldwin S, et al. 18FDG PET-CT imaging detects arterial inflammation and early atherosclerosis in HIV-infected adults with cardiovascular disease risk factors. J Inflamm (Lond) 2012;9:26. 
33. Knudsen A, Hag AM, Loft A, von Benzon E, Keller SH, Moller $\mathrm{HJ}$, et al. HIV infection and arterial inflammation assessed by (18)F-fluorodeoxyglucose (FDG) positron emission tomography (PET): a prospective cross-sectional study. J Nucl Cardiol 2015;22:372-80

34. Lawal IO, Ankrah AO, Popoola GO, Lengana T, Sathekge MM. Arterial inflammation in young patients with human immunodeficiency virus infection: A cross-sectional study using F-18 FDG PET/CT. J Nucl Cardiol 2019;26:1258-65.

35. Guaraldi G, Milic J, Prandini N, Ligabue G, Esposito F, Ciusa G, et al. (18)Fluoride-based molecular imaging of coronary atherosclerosis in HIV infected patients. Atherosclerosis 2020;297:127-35.

36. Zanni MV, Toribio M, Wilks MQ, Lu MT, Burdo TH, Walker J, et al. Application of a novel CD206+ macrophage-specific arterial imaging strategy in HIV-infected individuals. J Infect Dis 2017;215:1264-9.

37. Antinori A, Arendt G, Becker JT, Brew BJ, Byrd DA, Cherner M, et al. Updated research nosology for HIV-associated neurocognitive disorders. Neurology 2007;69:1789-99.

38. McArthur JC, Johnson TP. Chronic inflammation mediates brain injury in HIV infection: Relevance for cure strategies. Curr Opin Neurol 2020;33:397-404.

39. Rubin LH, Sacktor N, Creighton J, Du Y, Endres CJ, Pomper MG, et al. Microglial activation is inversely associated with cognition in individuals living with HIV on effective antiretroviral therapy. AIDS 2018;32:1661-7.

40. Vera JH, Guo Q, Cole JH, Boasso A, Greathead L, Kelleher P, et al. Neuroinflammation in treated HIV-positive individuals: A TSPO PET study. Neurology 2016;86:1425-32.

41. Garvey LJ, Pavese N, Politis M, Ramlackhansingh A, Brooks DJ, Taylor-Robinson SD, et al. Increased microglia activation in neurologically asymptomatic HIV-infected patients receiving effective ART. AIDS 2014;28:67-72.

42. Clifford DB, Fagan AM, Holtzman DM, Morris JC, Teshome M, Shah AR, et al. CSF biomarkers of Alzheimer disease in HIVassociated neurologic disease. Neurology 2009;73:1982-7.
43. Brew BJ, Pemberton L, Blennow K, Wallin A, Hagberg L. CSF amyloid beta42 and tau levels correlate with AIDS dementia complex. Neurology 2005;65:1490-2.

44. Mohamed M, Skolasky RL, Zhou Y, Ye W, Brasic JR, Brown A, et al. Beta-amyloid (Abeta) uptake by PET imaging in older HIV+ and HIV - individuals. J Neurovirol 2020;26:382-90.

45. Howdle GC, Quide Y, Kassem MS, Johnson K, Rae CD, Brew BJ, et al. Brain amyloid in virally suppressed HIV-associated neurocognitive disorder. Neurol Neuroimmunol Neuroinflamm 2020;7.

46. Cysique LA, Brew BJ. Vascular cognitive impairment and HIVassociated neurocognitive disorder: A new paradigm. J Neurovirol 2019;25:710-21.

47. Suri MFK, Zhou J, Qiao Y, Chu H, Qureshi AI, Mosley T, et al. Cognitive impairment and intracranial atherosclerotic stenosis in general population. Neurology 2018;90:e1240-7.

48. Daramola O, Ali H, McKenzie CA, Smith C, Benjamin LA, Solomon T. Pre-clinical atherosclerosis is found at post-mortem, in the brains of men with HIV. J Neurovirol 2021.

49. Benjamin LA, Corbett EL, Connor MD, Mzinganjira H, Kampondeni S, Choko A, et al. HIV, antiretroviral treatment, hypertension, and stroke in Malawian adults: A case-control study. Neurology 2016;86:324-33.

50. McLaughlin MM, Ma Y, Scherzer R, Rahalkar S, Martin JN, Mills $\mathrm{C}$, et al. Association of viral persistence and atherosclerosis in adults with treated HIV infection. JAMA Netw Open 2020;3:

51. Tawakol A, Ishai A, Li D, Takx RA, Hur S, Kaiser Y, et al. Association of arterial and lymph node inflammation with distinct inflammatory pathways in human immunodeficiency virus infection. JAMA Cardiol 2017;2:163-71.

Publisher's Note Springer Nature remains neutral with regard to jurisdictional claims in published maps and institutional affiliations. 